\title{
Study on the Influence of Non-electrical Parameters on Processing Quality of WEDM-HS and Improvement Measures
}

\author{
Guangyao Xiong ${ }^{1}$, Meizhu Zheng ${ }^{1}$, Deying $\mathrm{Li}^{1}$, Longzhi Zhao ${ }^{1}$, \\ Yanlin Wang ${ }^{1}$, and Minghui $\mathrm{Li}^{2}$ \\ ${ }^{1}$ School of Mechanical and Electrical Engineering, East China Jiaotong University, \\ Nanchang, Jiangxi, 330013, China \\ ${ }^{2}$ Department of Plastic Forming, Shanghai Jiaotong University, \\ Shanghai, 200030, China \\ xiongguangyao@163.com, zhengmeizhu@163.com, \\ lideyingecjtul163.com, zhaolongzhi@163.com, \\ WYL1921@yahoo.com.cn, liminghuia163.com
}

\begin{abstract}
The high-speed wire electrical discharge machining (WEDM-HS) machine has been more and more favoured by customers for the low manufacturing cost, the degree of automation and the advantages over the WEDM-LS in price performance ratio. However, the processing quality (the precision of form and position and the surface quality) of WEDM-HS is poorer than that of WEDM-LS, and the processing quality is one of the main technical indicators of WEDM. There are many factors on the processing quality of WEDM-HS, the effects of non-electrical parameters, including the machine precision, the wire-moving system and wire-moving way of the wire electrode and the working solution, on the processing quality of WEDM-HS are discussed, and the corresponding measures of improving the processing quality are put forward, which provide the sufficient basis for further improving the processing quality of WEDM-HS.
\end{abstract}

Keywords: WEDM-HS, Non-electrical parameters, Processing quality, Improvement measure.

\section{Introduction}

The wire electrical discharge machining (WEDM) is the method of EDM that the metal wire is used as tool-electrode [1]. In the processing, the anode of pulse power connects with the workpiece and the cathode connects with the electrode wire, the high-temperature generated by impulsive discharge between the workpiece and the wire electrode will make the workpiece melted and gasified to achieve processing, and the relative trajectory and velocity between them are controlled to cut out the workpiece with a certain shape and size. WEDM could be used to solve the processing problems of materials with special mechanical properties, such as high melting point, high hardness and ductility, and the parts will be with special structure and requirements, which are difficult or unsolvable for the traditional process. Therefore, it has the 
utmost importance in mold, forming tool, unmanageable materials and complex precision parts.

According to the moving way of wire electrode, WEDM machine can be divided into high-speed and low-speed CNC ones, which have great difference in structure and processing property $[2,3]$. Because the various schemes are taken in the high-speed and the low-speed WEDM, which leads to much difference in the structure of machines, the wire-moving systems and processing conditions, it may not be very appropriate to make a comparison between the processing performances of two machines one by one. Excluding the factor of cost, the accuracy, functionality, reliability, and processing stability of the domestic WEDM-HS machine have large gap comparing with foreign advanced WEDM-LS technology. In this paper, the ways of improving the processing surface quality of WEDM-HS will be discussed from the influence of main non-electric parameters on processing quality.

\section{Main Non-electrical Parameters on Processing Quality of WEDM-HS and Improvement Measure}

\subsection{Influence of the Precision of WEDM-HS on Processing Quality}

The precision of WEDM-HS is the primary condition to ensure the processing quality, and the precision of worktable is primarily decided by the precision of transmission parts (screw mandrel, nut and gear), the fit clearance, the equipping precision, and the work environment of machine et al. The processing quality is poor for the poor transmission precision of worktable. Therefore, the manufacturing precision of WEDM-HS should be strictly controlled to improve processing precision.

\subsection{Influence of the Stability of Wire-Moving System on Processing Quality and Control Measures}

The flexible metal wire is used as the electrode in WEDM, which is carried out by the role of impulsive discharge between the workpiece and wire electrode. The wire electrode will be pushed away from the discharge area, and bended backward with a certain flexivity when the wire electrode is discharging. As shown in Fig.1, line 1 is the actual track of wire electrode, the processing direction of which is L1; line 2 is the theoretical track of wire electrode, and line 3 is the actual track of wire electrode, the processing direction of which is L3. Under the condition of limiting flexivity, the dynamic WEDM-HS process is always simplified to the static variation, and the bending section of the wire electrode is approximated as an arc, so the actual discharge point has deviated $\mathrm{y} \mathrm{cm}$ from the theoretical cutting point, and the flexivity could be expressed as the following Eq.1 [1, 4].

$$
y=\frac{2 f L-f H}{4 F}
$$

Where $y$ is the flexivity $(\mathrm{cm}) ; L$ is the span length of wire electrode $(\mathrm{cm}) ; H$ is the thickness of work piece $(\mathrm{cm}) ; f$ is the discharge force and $F$ is the tensile force of wire electrode. 
The traditional wire cutting drives the wire electrode in V-groove of guide wheel to move back and forth through the rotation of guide wheel, the schematic diagram of guide wheel positioning as shown in Fig.2. The bearing ball is easy to wear, and produce the radial traverse for the speed of guide wheel is $8000 \mathrm{n} / \mathrm{s}$, which makes the vibration of wire electrode [5]. The wire electrode moves generally back and forth with the speed of $10 \mathrm{~m} / \mathrm{s}$, as a result, the bottom of V-groove will be abraded, so the accurate positioning of wire electrode can not be guaranteed. When the cutting directions are changed, the direction of L1, L2, L3 and L4 as shown in Fig.1, the position of tangent point and the tension between wire electrode and guiding wheel are variable, resulting in the change of flexivity between y1 and y3. It is obviously that y1 is larger than $\mathrm{y} 3$, and $\mathrm{y} 2$ equals with $\mathrm{y} 4$. As the form and position of wire electrode changes, it is difficult to improve precision.

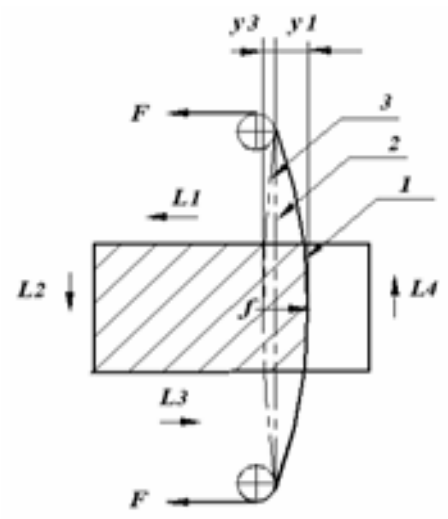

Fig. 1. Variations of form and position of wire electrode

(1-Processing track along L3; 2-Theoretical track; 3- Processing track along L1)
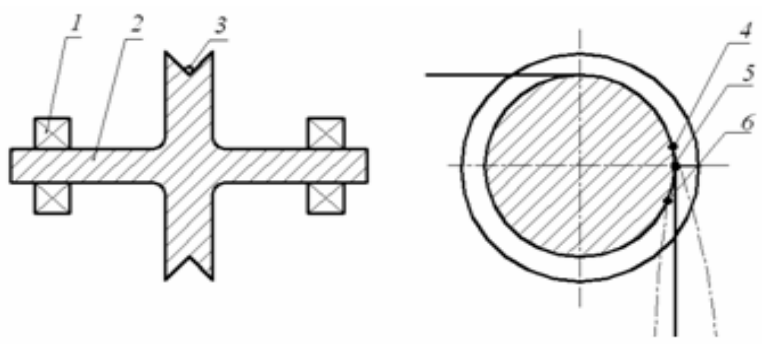

Fig. 2. The positioning of guide wheel

(1-Bearing; 2-Guide wheel; 3-Wire electrode; 4-Tangent point processing along L1;

5-Tangent point of theoretical track; 6-Tangent point processing along L3) 
The flexivity of wire electrode cased by the discharge power has no influence on processing precision when the straight line is cut, and the flaw is produced called sunken corner for processing the corner to influence the processing precision, the formation of sunken corner as shown in Fig.3, in addition, the vibration of wire electrode will inevitably produce the deviation of cylindricity when the cylinder is cut. Therefore, the form of spacial variations and the position of wire electrode should be steadied in order to reduce the accuracy error of wire electrode.

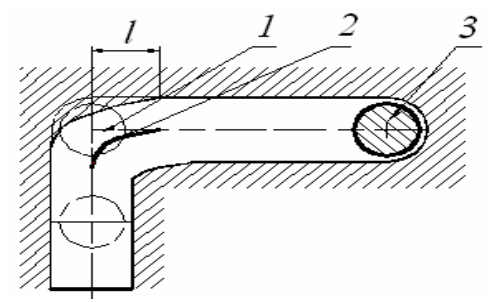

Fig. 3. The formation of sunken corner

(1-Theoreti-cal track; 2-Actual track; 3-Wire electrode)

In order to reduce the flexivity and steady on the variations of form and position of wire electrode, two guiders with wear properties are adopted to position the wire electrode up and down by Shanghai Mass Electronic Equipment Co., Ltd. The wire electrode passes through the guider as shown in Fig.4, which is blocked by the small holes of guider. The aperture and diameter of wire electrode match reasonably, and the deviation cased by wire electrode is blocked by the small holes when the processing directions are changed, which is the four directions. As the guide device is installed, the actual distance L between two fulcrums up and down of wire electrode is decreased. According to the Eq.1, the flexivity is actually reduced. So the variations of form and position of wire electrode are inhibited and steadied, in order to further improve the stability of processing precision.

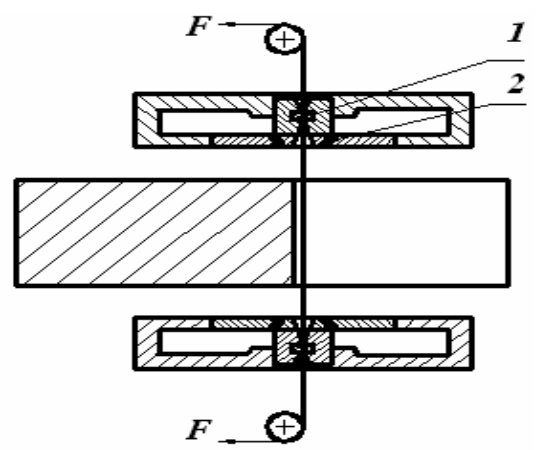

Fig. 4. The positioning of guiders with wear properties

(1-Guiders; 2-Spray nozzle plate) 


\subsection{Influence of the Movement Back and Forth of Electrode Wire and Working Solution on Processing Quality and Control Measures}

The workpiece surface processed by WEDM-HS will generally produce obvious stripes, when there is almost no stripe for WEDM-LS, it is decided by the cooling method and wire-moving way of WEDM-HS [6]. In WEDM-LS, the workpiece is immersed in de-ionized water to cool, and the wire-moving way is wire-moving one-way with low speed, while the rushing fluid is adopted to cool in WEDM-HS, the working solution is emulsion, and the wire-moving is instability to produce stripes inevitably, for the wire-moving way is wire-moving back and forth with high speed. The stripes of workpiece surface processed by WEDM-HS can generally be divided into two kinds, the one is the stripes with color, whose black and white concentration in the entrance and exit of wire-moving are different, and sometimes the kerf of exit side is slightly wider. The basic reason is the detergency of working solution. It is generally acknowledged that the stripes are caused by the rushing fluid and the dirty degree of working solution in entrance and exit. The other is the convex-concave stripes. When the wire electrode moves from top to down, the working solution is taken from the top into kerf along the wire electrode, and the discharge products are taken out in the processing zone from the bottom along wire electrode to accumulate at the exit of kerf, blocking the new working solution to come into kerf. So the insulated state of working solution is not normal at the exit of wire electrode, resulting in the abnormal discharge, and the kerf width of exit is less than that of the entrance as shown in Fig.5. When the wire electrode moves back and forth, the height of entrance and exit of wire electrode are different in the same surface, which will affect the processing precision and surface roughness of wire-cutting.

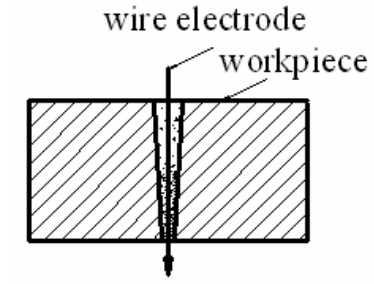

(a) Moving from top to down

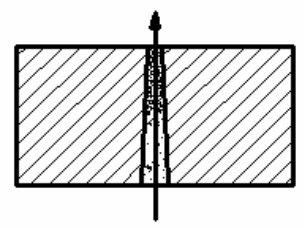

(b) Moving from down to up

Fig. 5. Wire-cutting back and forth

The measures to reduce strips as follows:

(1) To remove the black and white stripes, the saponification solution with strong detergency should be used as the working solution of WEDM, and the detergent state of kerf should be improved by adding some detergency substance. The spiral nozzle is used to spray, and the guiders with high wear properties mentioned above have reasonable spraying structure, making the working solution even eject along the axis of wire electrode. 
(2) The wire electrode of general WEDM-HS does not reverse until the entire wire go to end, in order to form the convex-concave stripes. The processing zone that the wire electrode passes through is longer, the convex-concave stripes are more obvious, whereas the stripes are few. When the electrode wire is short to a certain extent, the convex-concave stripes could be removed. In order to remove the convex-concave stripes, the key is to control the length of wire electrode in each direction and the repeated addition of cutting traces of wire electrode in processing zone, it's to remove the convex-concave stripes. The wire-moving method of ultra-short trip back and forth is adopted by Shanghai Mass Electronic Equipment Co., Ltd. In order to achieve the cutting without strip, the actual cutting distance of reversing direction each time is very short, it's about a quarter of the wire diameter, and the irregularities formed during the movement of wire electrode up and down are stacked with each other, so the black and white and convex-concave stripes will be difficult to find out. If the cutting path in reversing each time is shorter, the effect will be better.

The wire-cutting technology of ultra-short trip back and forth dose not use the short molybdenum wire, which will lead to the short section of wire electrode long-term concentrated to accelerate the loss of wire diameter and break of wire, and affect seriously the processing precision. Although the storing wire cylinder rotates and reverses frequently, the whole cutting process is still performed with the full-length molybdenum wire of storing wire cylinder, in order to avoid the loss of wire diameter after the concentrated discharge effectively. However, the high-frequency pulse power is cut off frequently to reverses frequently the storing wire cylinder, which will affect the average cutting speed.

\subsection{Influence of Residual Stress Inside the Workpiece on Processing Quality}

When the roughcast after heat treatment is processed by WEDM, due to the removal of much metal and interrupted cutting, the relative balance of residual stress inside the workpiece will be destroyed to reduce the processing precision of parts. Even the material will be suddenly cracked in the process of cutting, and the wire electrode is caught and pulled apart by kerfs in severe cases.

Therefore, materials with excellent harden ability and small deformation of heat treatment should be chosen. In addition, the heat processing methods should be chosen correctly and the standards of heat treatment should be performed strictly.

\section{Processing Quality}

The TP-25 WEDM machine produced by Shanghai Mass Electronic Equipment Co., Ltd. is used to process. The processing material is $\mathrm{Cr} 12$, which size is $10 \mathrm{~mm} \times 10$ $\mathrm{mm} \times 40 \mathrm{~mm}$. The processing condition and processing quality are shown in Table 1 .

The research results show that compared to the guide wheel to position and adopting the wire-moving of whole trip back and forth, the surface roughness value can be reduced $0.8 \mu \mathrm{m}$, the maximum error of sunken corner can be reduced $10 \mu \mathrm{m}$, and there is no reversing stripe using the guider with high wearing resistance to position and the wire-moving of ultra-short trip back and forth. So the processing quality of workpiece is greatly improved in spite of the decline of cutting speed. 
Table 1. The comparison of processing quality with different parameters

\begin{tabular}{ccccccc}
\hline Sample & $\begin{array}{c}\text { Position } \\
\text { mode }\end{array}$ & $\begin{array}{c}\text { Wire-moving } \\
\text { way }\end{array}$ & $\begin{array}{c}\text { Exist } \\
\text { reversing } \\
\text { strips or } \\
\text { not }\end{array}$ & $\begin{array}{c}\text { Surface } \\
\text { roughness } \\
(\mu \mathrm{m})\end{array}$ & $\begin{array}{c}\text { Max- } \\
\text { sunken } \\
\text { corner } \\
(\mu \mathrm{m})\end{array}$ & $\begin{array}{c}\text { Processing } \\
\text { time } \\
(\mathrm{s})\end{array}$ \\
\hline 01 & $\begin{array}{c}\text { By guide } \\
\text { wheel }\end{array}$ & $\begin{array}{c}\text { Whole trip } \\
\text { back and } \\
\text { forth } \\
\text { Ultra-short } \\
\text { trip back and } \\
\text { forth }\end{array}$ & exist & 2.4 & 17 & 3062 \\
\hline
\end{tabular}

\section{Conclusion}

1) The manufacturing precision of WEDM has great influence on the processing quality, and should be strictly controlled to improve machining precision.

2) The variations of form and position of electrode wire, producing inevitably in the process of WEDM-HS, which could be effectively controlled by the guiding device with high wearing resistance to improve the processing quality, if the wire-moving cutting method of ultra-short trip back and forth is applied to cut with no strips, however, the cutting speed will decline.

3) The strips could be reduced to improve the surface quality by using the working solution with the strong detergency and the appropriate spray method.

Acknowledgment. Work was supported by the National Natural Science Foundation of China (Grant No. 10972030).

\section{References}

1. Li, M.H.: Theoretical basis of EDM (In Chinese). National Defence Industry, Beijing (1989)

2. Li, M.H.: The Research Status and Development Trend of Electric Discharge Wire-cutting Technology. Die and Mould Technology (6), 49-52 (2002)

3. Guo, L.E., Liu, Z.X., Xing, X.F.: Study on dynamic stability of multiple cut on machine tool in HS WEDM. Electromachining (6), 22-25 (1999)

4. Li, M.Q., Li, M.H., Xiong, G.Y.: Study on the Variations of Form and Position of the Wire Electrode in WEDM-HS. International Journal of Advanced Manufacturing Technology 5(9), 929-934 (2005)

5. Xia, W.F.: Electric Sparkle Cutting Bad Reasons for Product Quality and Prevention. Equipment Manufacturing Technology (7), 178-182 (2009)

6. Liu, Z.D.: Technology Research of WEDM Using Composite Cooling Liquid. Electromachining \& Mould (12), 24-30 (2008) 